CARDIOVASCULAR MEDICINE

\title{
Improvement of stress LVEF rather than rest LVEF after coronary revascularisation in patients with ischaemic cardiomyopathy and viable myocardium
}

\author{
V Rizzello, D Poldermans, E Biagini, A F L Schinkel, R van Domburg, A Elhendy, E C Vourvouri, \\ M Bountioukos, A Lombardo, B Krenning, J R T C Roelandt, J J Bax
}

Heart 2005;91:319-323. doi: 10.1136/hrt.2004.037119

See end of article for authors' affiliations

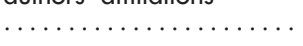

Correspondence to: Dr Don Poldermans Department of Cardiology, Thoraxcentre Room Ba 300, Erasmus MC, Dr Molewaterplein 40, 3015 GD Rotterdam, Netherlands;

d.poldermans@

erasmusmc.nl

Accepted 10 May 2004

\begin{abstract}
Objective: To evaluate prospectively the response of left ventricular ejection fraction (LVEF) to high dose dobutamine infusion in patients showing substantial viability, with and without improved resting LVEF after revascularisation.

Methods: Before and 9-12 months after revascularisation, 50 patients with ischaemic cardiomyopathy (LVEF $32(8) \%$ ) and substantial myocardial viability ( $\geqslant 4$ viable segments) underwent radionuclide ventriculography and dobutamine stress echocardiography. Patients were divided into group 1, patients with, and group 2, patients without significant improvement in resting LVEF ( $\geqslant 5 \%$ by radionuclide ventriculography) after revascularisation. The response of LVEF during dobutamine stress echocardiography was compared in these two groups.

Results: Groups 1 and 2 were comparable in baseline characteristics, resting LVEF, and number of viable segments (mean (SD) $7(4) \vee 6$ (2), not significant). After revascularisation, the LVEF response during dobutamine stress echocardiography improved significantly in both groups (group 1, 34 (10)\% to 56 (8)\%; group 2, 32 (10)\% to 46 (11)\%; both $p<0.001)$. Interestingly, although resting LVEF did not improve in group 2, peak stress LVEF after revascularisation did ( $p<0.001)$. Group 1 patients had, however, a greater increase in peak stress LVEF (group 1, 22 (10)\%; group 2, 13 (9)\%; $p<0.01$ ). New York Heart Association and Canadian Cardiovascular Society classes decreased in both groups.

Conclusions: Although patients with viable myocardium did not always have improved rest LVEF after revascularisation, peak stress LVEF improved. Assessment of improvement of resting function may not be the ideal end point to evaluate successful revascularisation.
\end{abstract}

A ssessment of myocardial viability is important in the management of patients with ischaemic cardiomyopathy. In patients with a substantial amount of viable myocardium, left ventricular (LV) dysfunction is likely to improve after coronary revascularisation. ${ }^{1}$ Improvement of resting LV ejection fraction (LVEF) has often been used to assess the success of coronary revascularisation of viable myocardium. ${ }^{1-8}$ Previous studies have reported a variable proportion of patients with viable myocardium with improved LVEF after revascularisation, ranging from 36$88 \% .^{35}$ Hence, resting LVEF does not always improve after revascularisation despite the presence of substantial myocardial viability. It has been suggested that contractile reserve may improve during inotropic stimulation after revascularisation even though resting function does not improve. ${ }^{9}{ }^{10}$ However, information about peak stress LVEF (as a marker of cardiac stress performance) after revascularisation is lacking. In particular, whether patients with viable myocardium who do not have improved resting LVEF may have improved peak stress LVEF after revascularisation is unknown. In the present study, combined low and high dose dobutamine stress echocardiography (DSE) was performed before and after revascularisation to evaluate postoperative changes in stress LVEF in patients with and without improvement in resting LVEF.

\section{METHODS}

\section{Study population}

The study population consisted of 56 patients ( 44 men, mean (SD) age 60 (11) years) with ischaemic cardiomyopathy
(LVEF $32(8) \%$ ) and a substantial amount of viable myocardium ( $\geqslant 4$ segments, $\geqslant 25 \%$ of the $\mathrm{LV}$ ) who were already scheduled for coronary revascularisation. Seven of these patients had taken part in a previous study. ${ }^{11}$ All patients had heart failure symptoms (mean (SD) New York Heart Association (NYHA) class 3.1 (0.7)), and 70\% had accompanying angina pectoris (Canadian Cardiovascular Society (CCS) class $2.5(0.6))$. A history of myocardial infarction was present in 54 patients (96\%). In these patients, myocardial infarction had occurred $\geqslant 6$ months before the study (median three years, range 0.7-22 years). The decision for revascularisation was based on clinical grounds (symptoms, presence or absence of ischaemia, and angiographic findings). Patients with severe (grade 3 to 4) mitral regurgitation were not included. Revascularisation was performed by coronary artery bypass grafting in 45 patients $(80 \%)$ and by angioplasty in 11 patients $(20 \%)$. None of the patients had a perioperative myocardial infarction. Two patients $(4 \%)$ died during the postoperative period (within 30 days) and four patients (one treated by angioplasty and three treated by bypass surgery) were excluded because of incomplete revascularisation according to the procedure report. Therefore, the final study population consisted of 50 patients (40 men, mean (SD) age 61 (11) years). These patients had complete revascularisation and were stable during the study period.

Abbreviations: CCS, Canadian Cardiovascular Society; DSE, dobutamine stress echocardiography; LV, left ventricular; LVEF, left ventricular ejection fraction; NYHA, New York Heart Association; RNV, radionuclide ventriculography 
Table 1 Baseline characteristics

\begin{tabular}{|c|c|c|c|}
\hline & $\begin{array}{l}\text { Group 1: improvers } \\
(n=26)\end{array}$ & $\begin{array}{l}\text { Group 2: non-improvers } \\
(n=24)\end{array}$ & p Value \\
\hline Men & $22(85 \%)$ & $18(75 \%)$ & NS \\
\hline Age (years) & $62(12)$ & $60(12)$ & NS \\
\hline Previous MI & $24(92 \%)$ & $24(100 \%)$ & NS \\
\hline Q wave $M I$ & $20(83 \%)$ & $21(87) \%$ & NS \\
\hline Anterior MI & 14 (54\%) & 15 (62\%) & NS \\
\hline NYHA class & $3.15(0.8)$ & $2.96(0.7)$ & NS \\
\hline CCS class & $2.57(0.7)$ & $2.67(0.6)$ & NS \\
\hline Stenotic vessels & $2.4(0.7)$ & $2.7(0.6)$ & NS \\
\hline Smoking & $12(46 \%)$ & $14(58 \%)$ & NS \\
\hline Hypertension & $22(85 \%)$ & $21(87 \%)$ & NS \\
\hline Hypercholesterolaemia & $16(61 \%)$ & $13(54 \%)$ & NS \\
\hline Family history of CAD & $13(50 \%)$ & $16(67 \%)$ & NS \\
\hline Diabetes mellitus & $1(5 \%)$ & $2(8 \%)$ & NS \\
\hline Lipid lowering drugs & $13(50 \%)$ & $10(42 \%)$ & NS \\
\hline ACE inhibitors & $17(65 \%)$ & $19(79 \%)$ & NS \\
\hline Nitrates & 22 (85\%) & 19 (79\%) & NS \\
\hline$\beta$ Blockers & $16(61 \%)$ & $16(67 \%)$ & NS \\
\hline Diuretics & $12(46 \%)$ & $16(67 \%)$ & NS \\
\hline Digoxin & $4(15 \%)$ & $7(29 \%)$ & NS \\
\hline Aspirin/anticoagulant & $26(100 \%)$ & $22(92 \%)$ & NS \\
\hline $\operatorname{LVEF}(\%)^{*}$ & $33(10)$ & 32 (7) & NS \\
\hline LV end diastolic volume (ml) & $160(59)$ & $194(39)$ & $<0.05$ \\
\hline LV end systolic volume (ml) & 105 (49) & $135(37)$ & $<0.05$ \\
\hline LV sphericity indext & $0.58(0.09)$ & $0.61(0.11)$ & NS \\
\hline \multicolumn{4}{|c|}{$\begin{array}{l}\text { Data are mean (SD) or number (\%). } \\
\text { *Assessed by radionuclide ventriculography. } \\
\text { tDerived by the ratio of left ventricular (LV) short to long axis dimensions in the apical four chamber view. }{ }^{15} \\
\text { ACE, angiotensin converting enzyme; CAD, coronary artery disease; CCS, Canadian Cardiovascular Society } \\
\text { LVEF, left ventricular ejection fraction; MI, myocardial infarction; NS, not significant; NYHA, New York Heart } \\
\text { Association. }\end{array}$} \\
\hline
\end{tabular}

\section{Study protocol}

The study protocol was prospectively designed to evaluate the response of LVEF to dobutamine challenge before and after revascularisation. DSE was performed one week before and 9-12 months after revascularisation. Radionuclide ventriculography (RNV) was also performed before and 9-12 months after revascularisation to assess improvement of resting LVEF by an independent technique. $\beta$ Blockers were not discontinued before DSE and RNV. An improvement in LVEF $\geqslant 5 \%$ after revascularisation was considered clinically significant. ${ }^{5}$ According to the presence or absence of significant improvement in resting LVEF after revascularisation, the patients were divided into two groups: group 1, patients with improved resting LVEF; and group 2, patients without improved resting LVEF. Next, the response of LVEF during dobutamine stress before and after revascularisation was compared in these two groups. In addition, heart failure symptoms and angina score were evaluated during the study period. The local ethics committee approved the protocol and all patients gave informed consent to participate in the study.

\section{Assessment of LVEF}

Before and 9-12 months after revascularisation, RNV was performed to assess LVEF improvement by an independent technique. RNV was performed at rest with the patient in the supine position after the administration of $740 \mathrm{MBq}$ of ${ }^{99 \mathrm{~m}}$ technetium. Images were acquired with a small field of view gamma camera (Orbiter; Siemens Corp, Iselin, New Jersey, USA) oriented in the $45^{\circ}$ left anterior oblique position with a $5-10^{\circ}$ caudal tilt. LVEF was calculated from the $45^{\circ} \mathrm{left}$ anterior oblique view by an automated technique. An improvement in LVEF $\geqslant 5 \%$ after revascularisation was considered clinically significant. ${ }^{5}$

\section{Dobutamine stress echocardiography}

All echocardiograms were recorded by commercially available equipment (Sonos 5500; Hewlett Packard, Philips Medical Systems, Eindhoven, the Netherlands) with a second harmonic 1.8-3.6 MHz transducer to optimise endocardial border visualisation. Standard parasternal and apical views of the LV were obtained ${ }^{12}$ at rest and at the end of each step of dobutamine infusion. Dobutamine was administered intravenously as previously described, ${ }^{13}$ starting at a dose of $5 \mu \mathrm{g}$ / $\mathrm{kg} / \mathrm{min}$ for five minutes, followed by $10 \mu \mathrm{g} / \mathrm{kg} / \mathrm{min}$ for five minutes. Subsequently, incremental dobutamine doses of $10 \mu \mathrm{g} / \mathrm{kg} / \mathrm{min}$ were given at three minute intervals up to a maximum dose of $40 \mu \mathrm{g} / \mathrm{kg} / \mathrm{min}$. Atropine (up to $2 \mathrm{mg}$ ) was administered intravenously if the test end point was not reached. Blood pressure, cardiac rhythm, and ST segment were continuously monitored.

Test end points were achievement of target heart rate, extensive new wall motion abnormalities, horizontal or down sloping ST segment depression ( $\geqslant 2 \mathrm{~mm}$ compared with baseline), severe angina, systolic blood pressure fall $>40 \mathrm{~mm} \mathrm{Hg}$, blood pressure > 240/120 mm Hg, and significant supraventricular or ventricular arrhythmia. Metoprolol (1-5 mg intravenously) was available to reverse the effects of dobutamine. Severely dysfunctional segments (including severe hypokinesia, akinesia, and dyskinesia) were evaluated for the presence of viability. Segments with a sustained improvement in wall motion up to high dose dobutamine and segments with a biphasic response or worsening of wall motion during DSE were considered viable. ${ }^{5}$ Segments with unchanged wall motion and segments with akinesia becoming dyskinesia were considered nonviable. $^{5}$ A substantial amount of viable myocardium was defined as the presence of $\geqslant 4$ viable segments. ${ }^{5}$ This definition is based on previous work with receiver operating characteristic curve analysis showing that recovery of function may be predicted in the presence of $\geqslant 4$ viable segments. $^{5}$

\section{Assessment of response in LVEF to high dose dobutamine}

The study results were analysed off line. LV volumes were measured at rest and during low and high dose dobutamine 
infusion with the biplane disk method, a modification of Simpson's rule. ${ }^{12}$ Subsequently, the LVEF at baseline and during low and high dose dobutamine were calculated by using the following equation: [end diastolic volume - end systolic volume]/end diastolic volume. All measurements were taken by an independent, experienced reader blinded to the clinical data and time of the study. Interobserver and intraobserver variability for LVEF calculation were reported previously (3.3\% and $5.3 \%$ respectively). ${ }^{14}$

\section{Assessment of functional status}

Before and after revascularisation, an independent physician blinded to all data conducted structured clinical interviews to assess the functional status according to the NYHA (for symptoms of heart failure) and the CCS (for angina) criteria.

\section{Statistical analysis}

Continuous data are expressed as mean (SD) and dichotomous data as proportions. Continuous data were compared by Student's $t$ test for paired and unpaired samples and two way analysis of variance to evaluate differences across time and between different groups, as indicated. Proportions was compared by $\chi^{2}$ analysis. For all tests, $p<0.05$ was considered significant.

\section{RESULTS}

Improvement of resting LVEF after revascularisation After revascularisation, resting LVEF improved significantly $(\geqslant 5 \%)$ in 26 patients (group 1) whereas it failed to improve in 24 patients (group 2). In particular, resting LVEF increased on average from $33(10) \%$ to $43(10) \%(p<0.001)$ in group 1 , whereas a slight but significant decrease was observed in group 2 (from $32(7) \%$ to $30(7) \%, p<0.05$ ). Baseline clinical characteristics of patients with (group 1) and without (group 2) improved resting LVEF were comparable (table 1). ${ }^{15}$ Resting LVEF by RNV and LV sphericity index were similar in the two groups (table 1), whereas LV end diastolic and end systolic volumes were significantly larger in group 2 (table 1 ). In addition, group 1 and group 2 were comparable in the extent of viable myocardium (7.4 (4) and 6.0 (2) segments, not significant) but group 2 patients had a larger extent of scar tissue (6.1 (2.3) v 3.7 (3.0), $\mathrm{p}<0.05)$.

\section{DSE before and after revascularisation}

The haemodynamic response during DSE was similar before and after revascularisation in the two groups. In particular, the peak rate-pressure product was 16.270 (2.895) $v 16.057$ (2.902) (not significant) in group $\mathrm{I}$ and 16.897 (3.226) $v$ 16.208 (3.225) (not significant) in group 2. The proportion of patients who reached $85 \%$ of the age predicted target heart rate was also similar before and after revascularisation (98\% $v$ $92 \%$, respectively, not significant). Before and after revascularisation, medication (including $\beta$ blockers) was comparable; only the use of nitrates was significantly reduced postoperatively $(85 \% v 19 \%, \mathrm{p}<0.001$, in group 1 and $79 \% v$

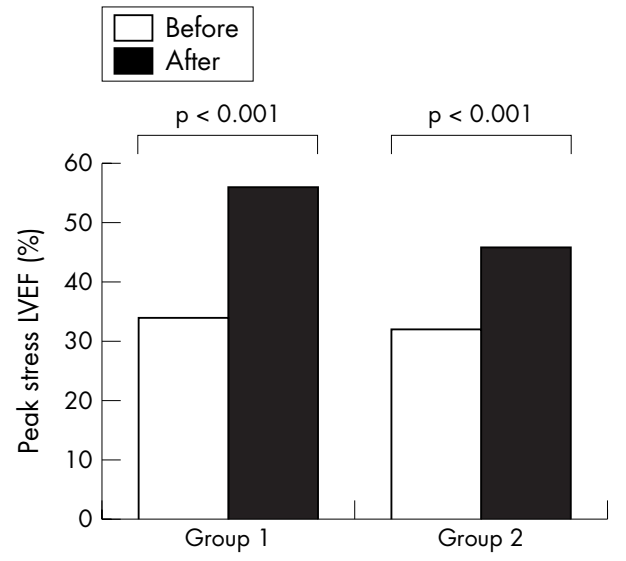

Figure 1 Bar graph showing the effect of revascularisation on peak stress left ventricular ejection fraction (LVEF) in viable patients with (group 1) and without (group 2) improvement of resting LVEF. After revascularisation, peak stress LVEF improved even in viable patients without improvement in resting LVEF.

$25 \%, \mathrm{p}<0.001$, in group 2). Table 2 shows the patterns of LVEF response during low and high dose DSE. Before revascularisation, LVEF had increased during low dose dobutamine infusion and deteriorated at peak DSE in both group 1 and group 2 ( $p<0.001$ by analysis of variance). The magnitude of change in LVEF during DSE was slightly higher in group 1 than in group $2(p=0.03$ by analysis of variance). After revascularisation, LVEF increased during low dose dobutamine infusion and remained increased at peak DSE in the two groups (both $\mathrm{p}<0.001$ by analysis of variance). The improvement in LVEF response during DSE after revascularisation was significant in both groups ( $p<0.001$ by analysis of variance) (table 2 ). In group 1 peak stress LVEF after revascularisation improved significantly compared with peak stress LVEF before revascularisation ( $\mathrm{p}<0.001$ ) (fig 1 ). Interestingly, after revascularisation, although resting LVEF had not improved in group 2, peak stress LVEF did also improve significantly in these patients $(\mathrm{p}<0.001)$ (fig 1). In particular, 19 of 24 patients $(79 \%)$ without improved resting LVEF had improved peak stress LVEF. The magnitude of improvement in LVEF at high dose dobutamine was higher in group 1 than in group $2(22(10) \%$ $v 13(9) \%, \mathrm{p}<0.01)$.

\section{Functional status}

After revascularisation all patients had a significant improvement in heart failure and angina symptoms (fig 2). In particular, the mean (SD) NYHA functional class improved from $3.2(0.8)$ to $1.8(0.7)$ in group $1(\mathrm{p}<0.001)$ and from $3.0(0.7)$ to $2.2(0.8)$ in group $2(\mathrm{p}<0.01)$. Similarly, the CCS class improved from $2.6(0.7)$ to $1.3(0.7)$ in group 1 and from $2.7(0.6)$ to $1.3(0.6)$ in group 2 (both $\mathrm{p}<0.001$ ).

Table 2 LVEF (\%) during dobutamine stress echocardiography

\begin{tabular}{|c|c|c|c|c|c|c|c|c|c|}
\hline & \multicolumn{4}{|c|}{ Before revascularisation* } & \multicolumn{4}{|c|}{ After revascularisation** } & \multirow[b]{2}{*}{ p Valuet } \\
\hline & Rest & LD & Peak & $\mathrm{p}$ Value & Rest & LD & Peak & $p$ Value & \\
\hline $\begin{array}{l}\text { Group } \\
1 \\
\text { Group }\end{array}$ & $34(8)$ & $46(11)$ & $34(10)$ & $<0.001$ & $43(9)$ & 54 (8) & $56(8)$ & $<0.001$ & $<0.001$ \\
\hline 2 & $31(8)$ & $41(9)$ & $32(10)$ & $<0.001$ & $31(7)$ & $44(10)$ & $46(11)$ & $<0.001$ & $<0.001$ \\
\hline
\end{tabular}




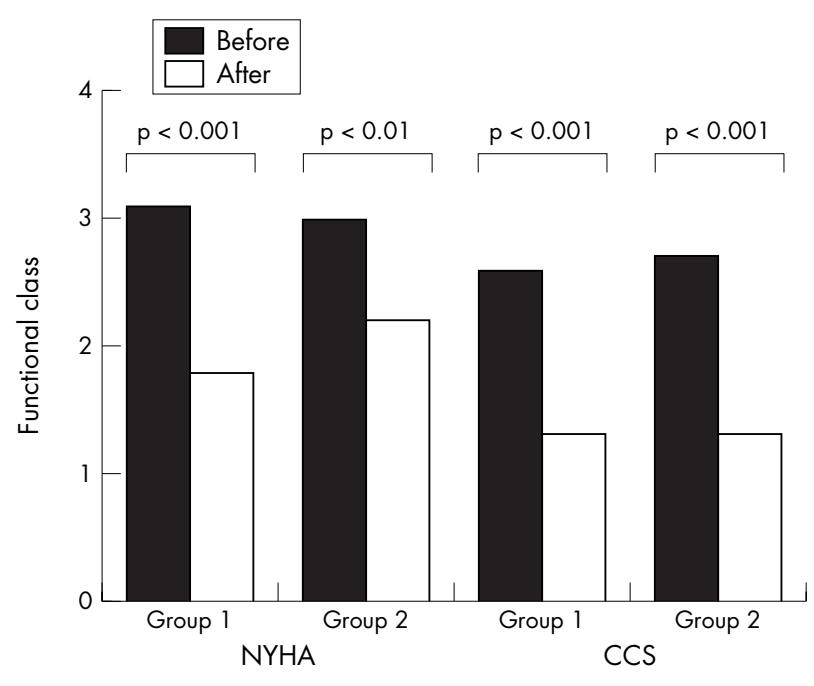

Figure 2 Bar graph showing the effect of revascularisation on heart failure and angina symptoms. After revascularisation, New York Heart Association (NYHA) and Canadian Cardiovascular Society (CCS) classes improved significantly both in patients with (group 1) and in patients without (group 2) improvement in resting LVEF.

\section{DISCUSSION}

Improvement of LVEF after coronary revascularisation has been described in variable proportions of patients with ischaemic cardiomyopathy and substantial myocardial viability. The findings in the present study showed that, although resting LVEF did not improve in $48 \%$ of patients with substantial viability, peak stress LVEF did improve compared with before revascularisation.

\section{Beneficial effect of coronary revascularisation on resting LV function}

Postoperative improvement of LV function has been considered the bench mark against which the preoperative methods to assess viability are measured. ${ }^{8}$ Initial studies showed that regional LV function improved after revascularisation, whereas in more recent studies global LV function improved. ${ }^{8}$ It has become clear that substantial myocardial viability is needed to obtain functional improvement. ${ }^{4-7}{ }^{16}$ Bax and colleagues $^{5}$ showed that LVEF improved in $82 \%$ of patients with substantial viable myocardium. However, other studies have shown that resting LVEF does not always improve after revascularisation. ${ }^{346-101416}$ In the present study, only patients with a substantial amount of viable myocardium (at least $25 \%$ of the LV) were included. After revascularisation, however, only $52 \%$ of the patients had significantly improved resting LVEF. Similarly, in the study by Pasquet and colleagues ${ }^{7}$ resting LVEF after revascularisation improved significantly in only $47 \%$ of patients shown by low and high dose DSE and nuclear imaging to have extensive viability. ${ }^{7}$ These varying percentages of patients experiencing recovery in LVEF after revascularisation are likely to be related to different characteristics of study populations. In particular, besides myocardial viability, additional factors may play a part in determining functional recovery after coronary revascularisation. It has been shown that the presence of extensive scar tissue may prohibit the increase of LVEF after revascularisation, despite viable myocardium. ${ }^{11}{ }^{17}$ Accordingly, in the present study the extent of scar tissue was larger in the patients who did not have improved LVEF. Also advanced LV remodelling may limit the improvement in LVEF despite the presence of viable myocardium. $^{2}{ }^{18-20}$ In the present study, baseline LV end diastolic and end systolic volumes were significantly larger in patients who did not have improved LVEF after revascularisation. Finally, delayed revascularisation and graft occlusion or restenosis after intervention may prevent functional recovery of viable myocardium..$^{21}$

\section{Effect of coronary revascularisation on LVEF response to dobutamine challenge}

In the present study, resting LVEF improved in only 52\% of patients, despite the presence of substantial viability. It has been suggested that, although resting function does not improve, the contractile reserve may increase during inotropic challenge after revascularisation. ${ }^{9}{ }^{10}$ In the study by Lombardo and colleagues, ${ }^{9}$ resting function did not improve after revascularisation in $57 \%$ of the dysfunctional regions shown by DSE to be viable before revascularisation. However, the contractile reserve was observed to increase during low dose dobutamine infusion in these regions after revascularisation. Elhendy and colleagues ${ }^{10}$ showed that LVEF during low dose dobutamine challenge increased even in patients who did not have improved resting LVEF after revascularisation. The responses of LVEF to high dose dobutamine after revascularisation (representing cardiac stress performance) have not been reported thus far.

In the present study, we evaluated the response of LVEF to combined low and high doses of dobutamine infusion in patients with (group 1) and without (group 2) improved resting LVEF after revascularisation. Before revascularisation, LVEF improved at low dose followed by a deterioration at high dose dobutamine (biphasic response). After revascularisation, although resting LVEF did not improve in $48 \%$ of patients with viable myocardium, peak stress LVEF did improve. Therefore, postoperative DSE identified additional patients who benefited from revascularisation in terms of global stress function, even though resting function did not improve. Although previous studies have shown that additional benefits (besides recovery of resting function) may be present after revascularisation, the present study uniquely showed that peak LVEF improved in patients without improved resting LVEF.

In a previous study that used a comparable low and high dose DSE protocol, Afridi and colleagues ${ }^{23}$ showed that the wall motion score index at peak stress also improved in patients without improved resting function (defined as an improvement of the wall motion score by $\geqslant 2$ grades in at least two contiguous segments). The findings in the present study are in line with this observation and show the beneficial effect of revascularisation on cardiac stress performance. Relief of ischaemia by restoration of the coronary flow reserve may be the mechanism responsible for maintaining the contractile function up to peak stress. This hypothesis is supported by data from Elhendy and colleagues $^{10}$ showing that the ischaemic score according to thallium-201 imaging decreased significantly after revascularisation in both patients with and patients without improved resting LVEF. Also, in the study by Afridi and colleagues, ${ }^{23}$ the majority of the improvement in stress wall motion score occurred in patients with evidence of ischaemia before revascularisation.

In line with previous studies, the observations in the present study suggest that assessment of resting LVEF after revascularisation may not be the ideal end point to evaluate the success of coronary revascularisation. Assessment of LVEF response to low and high dose DSE after revascularisation may be a more appropriate strategy to evaluate fully the benefit of revascularisation. The improvement of LVEF up to high dose dobutamine suggests the presence of a sustained contractile reserve and the absence of significant ischaemia. Preservation of contractile reserve and relief of ischaemia may be important in improving the prognosis of patients with 
ischaemic cardiomyopathy by preventing LV remodelling and ischaemia related arrhythmias. ${ }^{24}$

\section{Implications of improved stress function}

Post-revascularisation improvement of heart failure symptoms has been shown to relate to the presence of viable myocardium before revascularisation. ${ }^{592526}$ This was also observed in the current study. It is conceivable that improved stress LVEF may relate to improved functional class. It has been also shown that patients with myocardial viability who underwent revascularisation had a better prognosis than did medically treated patients. ${ }^{27}{ }^{28}$ This superior prognosis may also (in part) be related to the improvement of stress LVEF (reflecting absence of ischaemia). Further studies are needed to clarify this issue.

\section{Limitations}

Coronary angiography was not repeated after revascularisation, nor was perfusion imaging performed. Therefore, failure of resting LVEF to improve because of graft closure or restenosis after intervention cannot be excluded. However, none of the patients had an ischaemic response to DSE after revascularisation. The study population is relatively small. Further studies with more patients are needed to clarify the prognostic implications of these findings.

\section{Conclusion}

Assessment of resting LV function has been used as the yard stick to evaluate the success of coronary revascularisation in patients with ischaemic cardiomyopathy and viable myocardium. The findings in the present study showed that assessment of resting LVEF may underestimate the benefit of revascularisation, since stress LVEF may improve even in patients without improved resting LVEF. Moreover, the improvement in stress LVEF was accompanied by improved functional status.

\section{Authors' affiliations}

D Poldermans, E Biagini, A F L Schinkel, Ron van Domburg, A Elhendy, E C Vourvouri, M Bountioukos, B Krenning, J R T C Roelandt, Department of Cardiology, Thoraxcentre, Erasmus MC, Rotterdam, the Netherlands

V Rizzello, A Lombardo, Department of Cardiology, The Catholic University of the Sacred Heart, Rome, Italy

J J Bax, Department of Cardiology, Leiden University Medical Centre, Leiden, the Netherlands

\section{REFERENCES}

1 Wijns W, Vatner SF, Camici PG. Hibernating myocardium. N Engl J Med 1998;339:173-81.

2 Vanoverschelde JL, Gerber BL, D'Hondt AM, et al. Preoperative selection of patients with severely impaired left ventricular function for coronary revascularization: role of low-dose dobutamine echocardiography and exercise-redistribution-reinjection thallium SPECT. Circulation 1995;92:॥37-44.

3 Cuocolo A, Petretta M, Nicolai E, et al. Successful coronary revascularization improves prognosis in patients with previous myocardial infarction and evidence of viable myocardium at thallium-201 imaging. Eur J Nud Med 1998;25:60-8.

4 Ragosta M, Beller GA, Watson DD, et al. Quantitative planar restredistribution 201Tl imaging in detection of myocardial viability and prediction of improvement in left ventricular function after coronary bypass surgery in patients with severely depressed left ventricular function. Circulation 1993;87:1630-41.

5 Bax JJ, Poldermans D, Elhendy A, et al. Improvement of left ventricular ejection fraction, heart failure symptoms and prognosis after revascularization in patients with chronic coronary artery disease and viable myocardium detected by dobutamine stress echocardiography. J Am Coll Cardiol 1999;34: 163-9.
6 Pagano D, Bonser RS, Townend JN, et al. Predictive value of dobutamine echocardiography and positron emission tomography in identifying hibernating myocardium in patients with postischaemic heart failure. Heart 1998;79:281-8.

7 Pasquet A, Lauer MS, Williams MJ, et al. Prediction of global ventricular function after bypass surgery in patients with severe left ventricular dysfunction: impact of pre-operative myocardial function, perfusion, and metabolism. Eur Heart J 2000;21:125-36.

8 Bax JJ, Wijns W, Cornel JH, et al. Accuracy of currently available techniques for prediction of functional recovery after revascularization in patients with left ventricular dysfunction due to chronic coronary artery disease: comparison of pooled data. J Am Coll Cardiol 1997;30:1451-60.

9 Lombardo A, Loperfido F, Trani C, et al. Contractile reserve of dysfunctional myocardium after revascularization: a dobutamine stress echocardiography study. J Am Coll Cardiol 1997;30:633-40.

10 Elhendy A, Cornel JH, van Domburg RT, et al. Effect of coronary artery bypass surgery on myocardial perfusion and ejection fraction response to inotropic stimulation in patients without improvement in resting ejection fraction. Am J Cardiol 2000;86:490-4.

11 Rizzello V, Schinkel AF, Bax JJ, et al. Individual prediction of functional recovery after coronary revascularization in patients with ischemic cardiomyopathy: the scar-to-biphasic model. Am J Cardiol 2003;91:1406-9.

12 Schiller NB, Shah PM, Crawford M, et al. Recommendations for quantitation of the left ventricle by two-dimensional echocardiography. American Society of Echocardiography committee on standards, subcommittee on quantitation of two-dimensional echocardiograms. J Am Soc Echocardiogr 1989;2:358-67.

13 Salustri A, Ciavatti M, Seccareccia F, et al. Prediction of cardiac events after uncomplicated acute myocardial infarction by clinical variables and dobutamine stress test. J Am Coll Cardiol 1999;34:435-40.

14 Rocchi G, Poldermans D, Bax JJ, et al. Usefulness of the ejection fraction response to dobutamine infusion in predicting functional recovery after coronary artery bypass grafting in patients with left ventricular dysfunction. Am J Cardiol 2000;85:1440-4.

15 Otsuji Y, Kumanohoso T, Yoshifuku S, et al. Isolated annular dilation does not usually cause important functional mitral regurgitation: comparison between patients with lone atrial fibrillation and those with idiopathic or ischemic cardiomyopathy. J Am Coll Cardiol 2002;39:1651-6.

16 Cornel JH, Bax JJ, Elhendy A, et al. Biphasic response to dobutamine predicts improvement of global left ventricular function after surgical revascularization in patients with stable coronary artery disease: implications of time course of recovery on diagnostic accuracy. J Am Coll Cardiol 1998;31:1002-10.

17 Beanlands RS, Ruddy TD, deKemp RA, et al. Positron emission tomography and recovery following revascularization (PARR-1): the importance of scar and the development of a prediction rule for the degree of recovery of left ventricular function. J Am Coll Cardiol 2002;40:1735-43.

18 Vanoverschelde JL, Depre C, Gerber BL, et al. Time course of functional recovery after coronary artery bypass graft surgery in patients with chronic left ventricular ischemic dysfunction. Am J Cardiol 2000;85: 1432-9.

19 Nijland F, Kamp O, Verhorst PMJ, et al. Myocardial viability: impact on left ventricular dilatation after acute myocardial infarction. Heart 2002:87:17-22.

20 Schinkel AF, Poldermans D, Rizzello V, et al. Why do patients with ischemic cardiomyopathy and a substantial amount of viable myocardium not always recover in function after revascularization? J Thorac Cardiovasc Surg 2004; 127:385-90.

21 Bax JJ, Schinkel AF, Boersma E, et al. Early versus delayed revascularization in patients with ischemic cardiomyopathy and substantial viability: impact on outcome. Circulation 2003;108(suppl 1):1139-42.

22 Vanoverschelde JL, Pasquet A, Gerber B, et al. Pathophysiology of myocardial hibernation: implications for the use of dobutamine echocardiography to identify myocardial viability. Heart 1999;82(suppl 3):1II1-7.

23 Afridi I, Qureshi U, Kopelen HA, et al. Serial changes in response of hibernating myocardium to inotropic stimulation after revascularization: a dobutamine echocardiographic study. J Am Coll Cardiol 1997;30:1233-40.

$24 \mathrm{Kaul} \mathrm{S}$. There may be more to myocardial viability than meets the eye. Circulation 1995:92:2790-3.

25 Di Carli MF, Asgarzadie F, Schelbert HR, et al. Quantitative relation between myocardial viability and improvement in heart failure symptoms after revascularization in patients with ischemic cardiomyopathy. Circulation 1995;92:3436-44.

26 Eitzman D, al-Aouar Z, Kanter HL, et al. Clinical outcome of patients with advanced coronary artery disease after viability studies with positron emission tomography. J Am Coll Cardiol 1992;20:559-65.

27 Allman KC, Shaw $\amalg$, Hachamovitch R, et al. Myocardial viability testing and impact of revascularization on prognosis in patients with coronary artery disease and left ventricular dysfunction: a meta-analysis. J Am Coll Cardiol 2002;39: 1151-8.

28 Bourque JM, Hasselblad V, Velazquez EJ, et al. Revascularization in patients with coronary artery disease, left ventricular dysfunction, and viability: a meta-analysis. Am Heart J 2003;146:621-7. 